\title{
Impact of genetic profiles on the efficacy of anti-EGFR antibodies in metastatic colorectal cancer with $K R A S$ mutation
}

\author{
TOMOKAZU KISHIKI ${ }^{1}$, HIROAKI OHNISHI ${ }^{2}$, TADAHIKO MASAKI ${ }^{1}$, KOUKI OHTSUKA $^{2}$, \\ YASUO OHKURA $^{3}$, JYUNJI FURUSE ${ }^{4}$, MASANORI SUGIYAMA ${ }^{1}$ and TAKASHI WATANABE ${ }^{2}$ \\ Departments of ${ }^{1}$ Surgery, ${ }^{2}$ Laboratory Medicine, ${ }^{3}$ Pathology and ${ }^{4}$ Medical Oncology, \\ Kyorin University School of Medicine, Mitaka, Tokyo 181-8611, Japan
}

Received January 14, 2014; Accepted March 11, 2014

DOI: $10.3892 / o r .2014 .3179$

\begin{abstract}
Reports indicate that, even in $K R A S$-mutated colon cancer, there are subsets of patients who benefit from antiEGFR monoclonal antibody (MoAb) treatment. The aim of the present study was to identify genetic profiles that contribute to the responsiveness of metastatic colorectal cancer (mCRC) to anti-EGFR MoAb. We retrospectively evaluated the efficacy of anti-EGFR MoAb in mCRC patients with $K R A S$ mutations according to KRAS mutational subtypes, $B R A F$ and PIK3CA mutational status and PTEN and MET expression. Among 21 patients with KRAS-mutant tumors, 8 (38\%) harbored p.G13D, 7 (33\%) harbored p.G12V, 5 (24\%) harbored p.G12D, and $1(5 \%)$ harbored p.G12C mutation. Patients with the p.G13D mutation exhibited a significantly higher disease control rate than patients with other KRAS mutations $(\mathrm{P}=0.042)$, and tended to show a longer progression-free survival (PFS) than patients with other KRAS mutations with marginal significance $(\mathrm{P}=0.074)$. Patients with loss of PTEN had significantly shorter PFS than those with normal PTEN expression in patients with KRAS mutations $(\mathrm{P}=0.044)$. MET overexpression was significantly associated with shorter PFS compared to normal MET expression in patients with KRAS mutations $(\mathrm{P}=0.016)$. Our data demonstrated the potential utility of alterations in PTEN and MET expression as predictive markers for response to anti-EGFR MoAbs in mCRC patients with KRAS mutations. In addition, we confirmed the predictive value of the KRAS p.G13D mutation for better response to anti-EGFR therapies in comparison with other KRAS mutations.
\end{abstract}

Correspondence to: Dr Tomokazu Kishiki, Department of Surgery, Kyorin University School of Medicine, 6-20-2 Shinkawa, Mitaka, Tokyo 181-8611, Japan

E-mail: kishikitomokazu@yahoo.co.jp

Key words: colorectal cancer, KRAS mutation, MET, PTEN, anti-EGFR therapy

\section{Introduction}

During the last decade, several clinical studies have confirmed that targeting the epidermal growth factor receptor (EGFR) with specific monoclonal antibodies (MoAbs) may improve the outcome of metastatic colorectal carcinoma (mCRC) patients $(1,2)$. Mutations of the $K R A S$ oncogene, an important intracellular signaling molecule downstream of EGFR, have been identified as a strong negative predictor for response to anti-EGFR-based therapies (3-5). As a consequence, mutation testing of KRAS has become mandatory for the approval for the use of cetuximab and panitumumab in the treatment of mCRC by the US Food and Drug Administration (FDA), the European Medicines Agency (EMA), and the Japanese Ministry of Health, Labour and Welfare (MHLW) $(3,6,7)$. Hence, only mCRC patients with confirmed KRAS wild-type status are currently eligible for therapies using anti-EGFR antibodies in many countries.

On the other hand, reports indicate that, even in KRASmutated colon cancer, there are subsets of patients who benefit from anti-EGFR antibody treatment (8-10). These studies suggest that all KRAS mutations are not equivalent in their biological characteristics. First, an in vitro study revealed that cells with KRAS codon-13 mutations (mainly the p.G13D mutation) exhibit weaker transforming activity than those with codon-12 mutations $(3,11)$. Second, a subset of patients presenting with tumors with KRAS mutations, particularly the p.G13D mutation, responded to anti-EGFR treatment (8-10). Furthermore, in a recent meta-analysis using a pooled data set of 579 patients with chemotherapy-refractory colon carcinoma, the p.G13D mutation was reported to have predictive value for the treatment of mCRC with cetuximab (12). These findings indicate that anti-EGFR antibodies may have a stronger effect on tumors with the KRAS p.G13D mutation than those with other KRAS mutations. However, several studies did not detect a significant survival advantage in patients with p.G13D-mutated tumors treated with cetuximab monotherapy $(13,14)$. Therefore, the impact of the subtype of $K R A S$ mutation on responsiveness to anti-EGFR therapies remains uncertain.

A second critical aspect of EGFR-targeted therapies comes from the presence of other genetic abnormalities that can affect responses to such therapies in KRAS wild-type 
patients. Even in a KRAS wild-type group, less than $50 \%$ of patients responded to EGFR-targeted therapy $(4,5,15)$. These observations have prompted investigators to analyze the involvement in mCRC of other genes of the RAS/RAF/MAPK and PI3K/PTEN/Akt pathways. In this regard, the presence of oncogenic deregulation of EGFR and other members of its downstream signaling pathways, such as $B R A F, P I K 3 C A$, and PTEN, have been shown to influence the responsiveness to cetuximab and panitumumab and could, therefore, help to identify non-responder patients $(4,5,15-17)$. Furthermore, recent studies also have suggested that activation of MET, a tyrosine kinase that acts as a receptor for hepatocyte growth factor (HGF) and can activate the RAS/RAF/MAPK and PTEN/PI3K/Akt pathways, may be a novel mechanism of cetuximab resistance in CRC (18-20). However, no studies have yet addressed whether these additional aberrations may influence the responsiveness to anti-EGFR MoAb therapies in $K R A S$-mutated mCRC, mostly because these MoAb therapies are currently not recommended for this type of mCRC.

In the present study, we enrolled mCRC patients with $K R A S$ mutations who had been treated with anti-EGFR MoAbs before the start of such restrictions for this therapy. Retrospective analyses of these patients enabled us to evaluate the impact of KRAS mutation subtype, together with additional aberrations in other EGFR downstream genes, on the efficacy of anti-EGFR MoAb therapies in mCRCs with mutant KRAS. In addition to evaluating the association between the types of $K R A S$ mutations and the therapeutic efficacy of anti-EGFR therapies, we investigated whether PTEN or MET expression and $B R A F$ or PIK3CA mutation might influence the outcome in $K R A S$-mutant patients with mCRC treated by anti-EGFR MoAbs. Our final goal was to identify a group of patients which will benefit from such treatment among KRAS-mutant patients with mCRC.

\section{Patients and methods}

Patients. The clinical outcome of anti-EGFR MoAb therapy was retrospectively analyzed for possible associations with the molecular features of tumors in the mCRC patients. This study enrolled 81 Japanese patients who were treated at the Department of Gastroenterological Surgery and Medical Oncology, Kyorin University Hospital, between November 2008 and March 2012. All patients presented with histologically confirmed mCRC, and had been treated with salvage chemotherapy incorporating cetuximab or panitumumab. Clinical features of the patients and pathological profiles of the tumors were obtained from patient medical records. Cetuximab, as monotherapy or in combination with irinotecan, was administered intravenously (i.v.) at a loading dose of $400 \mathrm{mg} / \mathrm{m}^{2}$ over $2 \mathrm{~h}$, followed by weekly doses administered at $250 \mathrm{mg} / \mathrm{m}^{2}$ over $1 \mathrm{~h}$. Panitumumab was administered i.v. every 2 weeks at a dose of $6 \mathrm{mg} / \mathrm{kg}$. Treatment was continued until disease progression (PD) or toxicity occurred. Clinical evaluation and tumor response were analyzed according to Response Evaluation Criteria in Solid Tumors (RECIST) (21). Genetic alterations (subtype of KRAS mutation, mutations of $B R A F$ and PIK3CA, loss of PTEN expression and MET overexpression) were retrospectively investigated in patient tumor specimens as described below, and the association with the response to anti-EGFR MoAb therapies was analyzed in patients with mutant $K R A S$. This study was approved by the Research Ethics Committee of Kyorin University School of Medicine Hospital.

Mutational analysis of KRAS, BRAF and PIK3CA by direct sequencing. Paraffin-embedded tissues (primary or metastatic) were sectioned at $10-\mu \mathrm{m}$ thicknesses and mounted as three separate slides per tissue. The resulting slides were treated three times with xylene and then washed with ethanol. To minimize contamination by normal DNA, areas in which at least $70 \%$ of the cells exhibited disease-specific pathology were dissected under a binocular microscope; DNA was extracted from the dissected tissues using the QIAamp FFPE Tissue kit (Qiagen). Segments of the KRAS, BRAF and PIK3CA genes were amplified using gene-specific primers and subjected to direct DNA sequencing as previously described $(15,22)$. KRAS sequences were screened for point mutations in codons 12 and 13 within exon 2 , two hotspots that cumulatively include $>95 \%$ of mutations in this gene $(4,5,15)$. BRAF sequences were screened for V600E mutations within exon 15, a site at which $>95 \%$ of point mutations in this gene occur $(5,15)$. PIK3CA sequences were screened for mutations within exons 9 and 20, sites at which $>80 \%$ of point mutations in this gene occur (17).

\section{Immunohistochemistry of PTEN and MET. PTEN and MET} expression levels were evaluated by immunohistochemistry performed on $4-\mu \mathrm{m}$ tissue sections of paraffin-embedded specimens. PTEN was assessed using the 17.A mouse MoAb (1:25 dilution; Neomarkers, Thermo Fisher Scientific Inc., Fremont, CA, USA); MET was assessed using the SP44 rabbit MoAb (Spring Biosciences, Pleasanton, CA, USA) $(23,24)$. Negative controls were incubated with nonimmune solution instead of the primary antibody. Endothelial and hepatocellular carcinoma cells were used as positive controls for PTEN and MET expression, respectively. The PTEN and MET staining intensities were evaluated by a pathologist (Y.O.) who was blinded to the diagnosis of the individual patients.

To our knowledge, there currently are no validated scoring systems for interpretation of PTEN or MET staining intensity. Both PTEN and MET are localized primarily in the cytoplasm $(4,25)$; we therefore adopted a scoring system that has been used for other cytoplasmic proteins and is based on the intensity of immunoreactivity and the percentage of stained cells (26-28). Specifically, intensity was scored according to a four-tier system: 0, no staining; 1 , weak; 2 , moderate; and 3, strong. An additional 1,2, or 3 points were assigned depending in whether the percentage of positive cells was $<25 \%, 25-50 \%$ or $>50 \%$, respectively $(4,5)$.

We defined normal PTEN expression as a score of $\geq 4$ (Fig. 1A); scores of 0-3 were classified as loss of expression (Fig. 1B). We defined normal/low expression of MET as a score of 0-3 (Fig. 1C); scores of $\geq 4$ were classified as MET overexpression (Fig. 1D).

Statistical analysis. Comparison of categorical variables was performed with the $\chi^{2}$ test or the Fisher's exact test. The progression-free survival (PFS) and overall survival (OS) were calculated using the Kaplan-Meier method. Comparisons between different groups were performed using log-rank tests. 


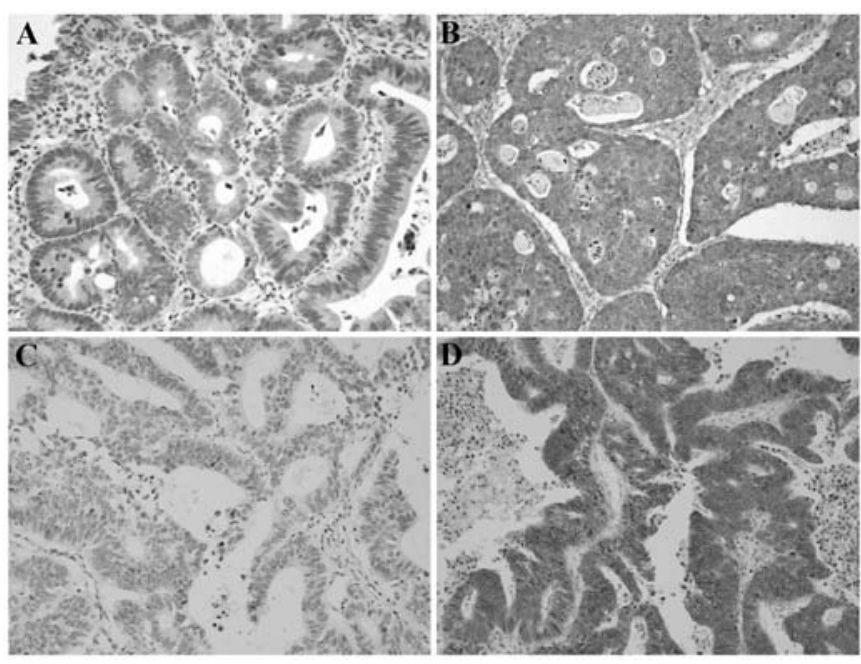

Figure 1. Representative examples of immunohistochemical staining in colorectal cancer. PTEN, loss of expression (A) and normal expression (B); MET, low expression (C) and overexpression (D).

Two-tailed P-values of $<0.05$ were considered significant. All analyses were performed using SPSS software (SPSS for Windows version 15.0; SPSS Inc., Chicago, IL, USA).

\section{Results}

Patient characteristics. Among the 81 patients enrolled in the study, 65 patients were able to be analyzed for all of the molecular parameters examined (mutations of KRAS, BRAF and PIK3CA, and expression of PTEN and MET). These 65 patients comprised 49 men and 16 women with a mean age of 68 years (range, 38 to 85 years). Among these 65 patients, the response rate (RR) and the disease control rate (DCR) were 23 and $51 \%$, respectively, and PFS and OS were 3.5 and 11.9 months, respectively.

The mutations in KRAS exon 2 were detected in $21(32 \%)$ of the 65 patients. Table I summarizes the characteristics of the 21 patients who harbored tumors with mutant KRAS genes. These 21 patients comprised 14 men and 7 women with a mean age of 67 years (range, 38 to 82 years). At a median follow-up of 5.2 months (range, 1.7-24.4 months), disease had progressed in all patients with KRAS mutations, and $16(70 \%)$ patients had died. In this 21-patient group, we observed no patients with complete response (CR) and partial response (PR), 3 with stable disease (SD) and 18 with progressive disease (PD). Therefore, the overall RR was $0 \%$, and the DCR was $14 \%$. $\mathrm{RR}$ and DCR were significantly lower in patients with KRAS mutations than in those with wild-type $K R A S$ : for RR, the values were 0 vs. $34 \%(\mathrm{P}<0.001)$; for $\mathrm{DCR}$, the values were 14 vs. $68 \%(\mathrm{P}<0.001)$. Median PFS and OS were significantly shorter in patients whose tumors carried KRAS mutations than in those without mutations (PFS: 1.8 vs. 5.9 months, $\mathrm{P}<0.001$; OS: 5.5 vs. 15.4 months; $\mathrm{P}=0.023)$.

Analysis of KRAS mutation subtypes. Among the 21 patients with KRAS-mutant tumors, 8 (38\%) harbored p.G13D, $7(33 \%)$ harbored p.G12V, 5 (24\%) harbored p.G12D, and $1(5 \%)$ harbored p.G12C mutation (Table I). Patients with the p.G13D
Table I. Characteristics of the CRC patients with mutant KRAS $(n=21)$.

\begin{tabular}{|c|c|c|}
\hline Characteristics & $\mathrm{n}$ & $\%$ \\
\hline \multicolumn{3}{|l|}{$K R A S$ mutation status } \\
\hline G13D & 8 & 38 \\
\hline G12V & 7 & 33 \\
\hline G12D & 5 & 24 \\
\hline G12C & 1 & 5 \\
\hline \multicolumn{3}{|l|}{ Age (years) } \\
\hline$\leq 70$ & 15 & 72 \\
\hline$>70$ & 6 & 28 \\
\hline \multicolumn{3}{|l|}{ Gender } \\
\hline Male & 14 & 65 \\
\hline Female & 7 & 35 \\
\hline \multicolumn{3}{|l|}{ Evaluated tumor } \\
\hline Primary & 20 & 95 \\
\hline Metastasis & 1 & 5 \\
\hline \multicolumn{3}{|l|}{ Stage at diagnosis } \\
\hline II and III & 8 & 38 \\
\hline IV & 13 & 62 \\
\hline \multicolumn{3}{|l|}{ Primary tumor location } \\
\hline Cecum & 1 & 5 \\
\hline Ascending colon & 2 & 10 \\
\hline Transverse colon & 1 & 5 \\
\hline Descending colon & 3 & 14 \\
\hline Sigmoid colon & 4 & 19 \\
\hline Rectum & 10 & 48 \\
\hline \multicolumn{3}{|l|}{ Tumor differentiation } \\
\hline Well/moderate & 21 & 100 \\
\hline Poor & 0 & 0 \\
\hline \multicolumn{3}{|l|}{ Site of metastasis } \\
\hline Liver & 18 & 86 \\
\hline Lung & 14 & 67 \\
\hline Peritoneum & 9 & 43 \\
\hline Others & 10 & 48 \\
\hline \multicolumn{3}{|l|}{ EGFR-targeted therapies } \\
\hline Cetuximab & 7 & 33 \\
\hline Cetuximab + irinotecan & 12 & 57 \\
\hline Panitumumab & 2 & 10 \\
\hline \multicolumn{3}{|l|}{$\begin{array}{l}\text { Anti-EGFR antibody } \\
\text { administration line }\end{array}$} \\
\hline $1 \mathrm{st}$ & 0 & 0 \\
\hline $2 \mathrm{nd}$ & 7 & 33 \\
\hline $3 r d$ & 9 & 43 \\
\hline 4 th or greater & 5 & 24 \\
\hline
\end{tabular}

mutation exhibited significantly higher DCR than patients with other KRAS mutations $(\mathrm{P}=0.042)$, but no significant difference in DCR compared to wild-type KRAS patients was noted ( $\mathrm{P}=0.124)$ (Table IIA). Regarding $\mathrm{RR}$, patients with 

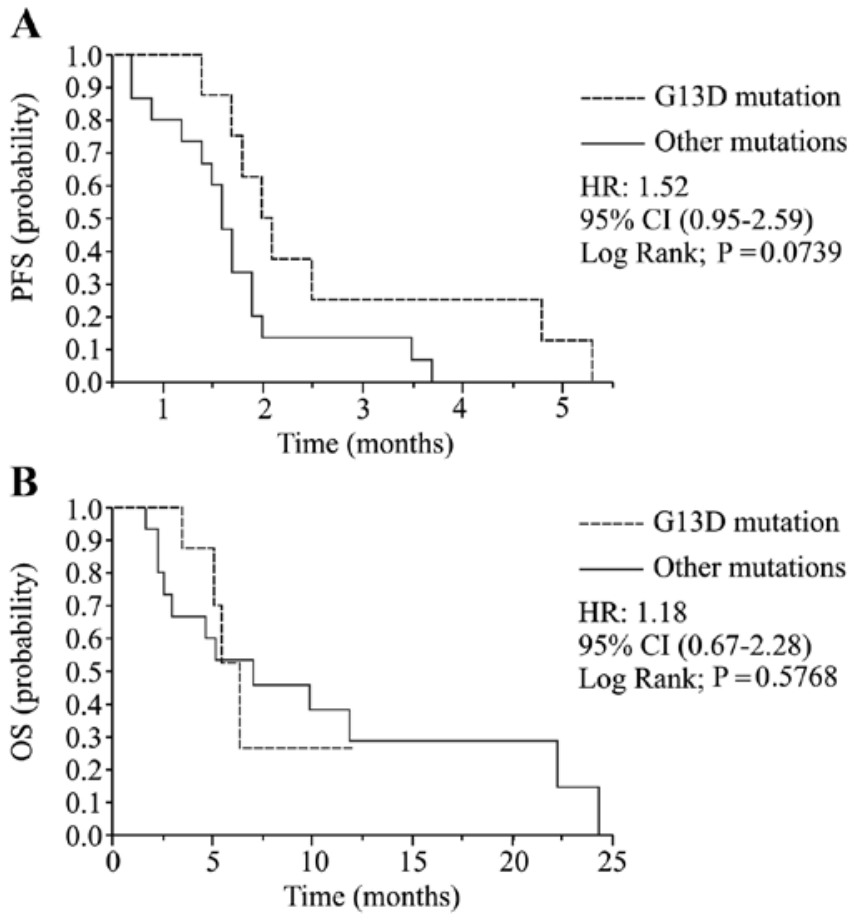

Figure 2. (A) Progression-free survival (PFS) in mutant KRAS patients classified according to the presence of p.G13D or other mutations. (B) Overall survival (OS) of patients classified according to the presence of p.G13D mutant $K R A S$ and/or other KRAS mutations.

p.G13D showed no significant difference compared to patients with other KRAS mutations or wild-type KRAS (Table IIA). Patients with the p.G13D mutation tended to have a prolonged PFS than patients with other KRAS mutations, and the difference was marginally significant. (2.1 vs. 1.7 months; HR, 1.52; 95\% CI, 0.95-2.59; P=0.074; Table IIB, Fig. 2A). No significant correlation was detected between the subtype of KRAS mutation and OS (Fig. 2B).

$B R A F$ and PIK3CA mutational analysis. As expected from the reported exclusivity between $K R A S$ and $B R A F$ mutations (15), $B R A F$ mutation was not detected among patients with $K R A S$ mutations; therefore, the impact of the $B R A F$ mutation could not be analyzed in this patient group (Table III). PIK3CA mutations were detected in $3(14 \%)$ of the 21 patients with KRAS mutations (Table III). No significant correlation was found between the PIK3CA mutational status and the subtype of KRAS mutation. None of the PIK3CA-mutant patients exhibited a response to anti-EGFR MoAb therapy. However, the difference in DCR between patients with and without PIK3CA mutation was not statistically significant $(\mathrm{P}=0.612$; Table IVA). PIK3CA mutations were not significantly associated with PFS (1.8 vs. 2.0 months; $\mathrm{P}=0.757)$ or OS (5.2 vs. 24.4 months; $\mathrm{P}=0.187$ ) (Table IVB, Fig. 3A and $\mathrm{B}$ ).

PTEN immunohistochemical evaluation. Nine (43\%) of the 21 patients with KRAS mutations exhibited loss of PTEN expression. No significant correlation was found between PTEN expression status and the subtype of KRAS mutation (Table III). Patients with loss of PTEN had significantly shorter PFS than those with normal PTEN expression (1.7 vs.
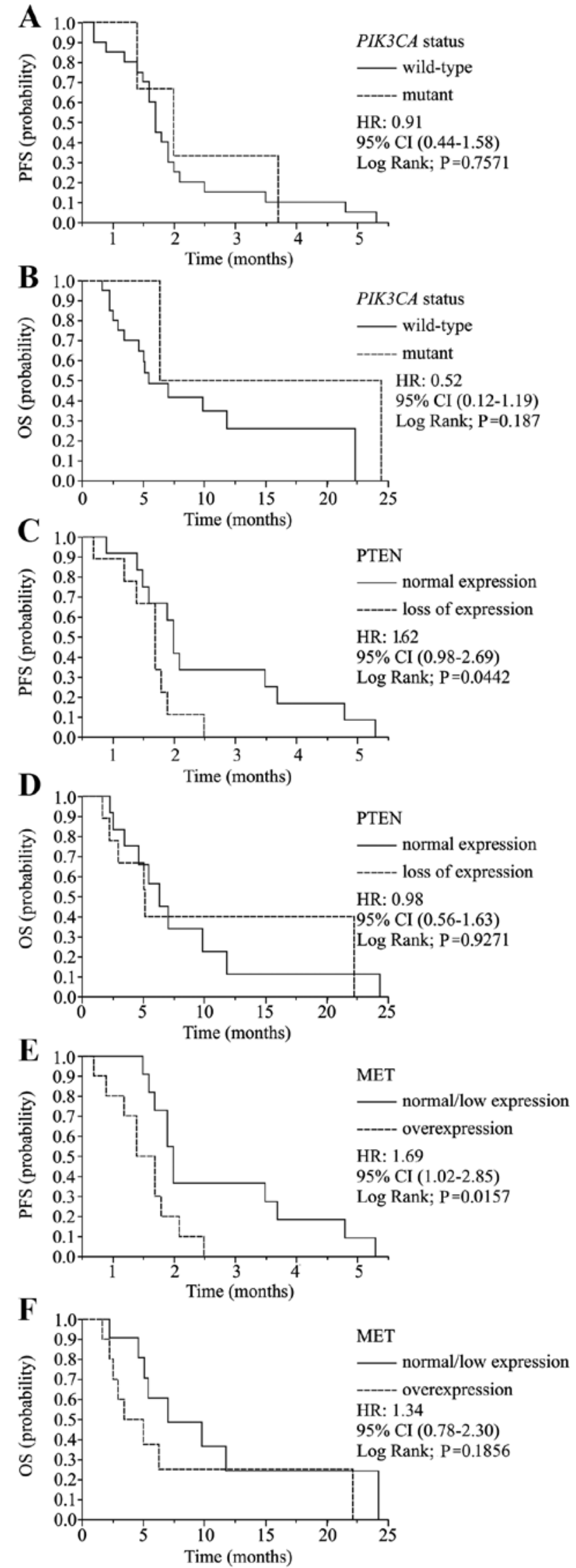

Figure 3. (A) Progression-free survival (PFS) and (B) overall survival (OS) in mutant KRAS patients according to PIK3CA mutational status. (C) Progression-free survival (PFS) and (D) overall survival (OS) in mutant KRAS patients classified according to PTEN expression status. (E) Progression-free survival (PFS) and (F) overall survival (OS) in mutant KRAS patients classified according to MET expression status. 
Table II. Effect of KRAS status on RR, DCR, PFS and OS.

A, Effect of KRAS status on RR and DCR

\begin{tabular}{lrrrrrrr}
\hline & $\mathrm{n}$ & $\mathrm{PR}$ & $\mathrm{SD}$ & $\mathrm{PD}$ & $\mathrm{RR}(\%)$ & $\mathrm{P}$-value & DCR (\%) \\
\hline KRAS & & & & & & & \\
G13D & 8 & 0 & 3 & 5 & 0 & 38 \\
Other mutations & 13 & 0 & 0 & 13 & 0 & $\mathrm{NA}^{\mathrm{a}}$ & 0 \\
Wild-type & 44 & 15 & 15 & 14 & 34 & $0.087^{\mathrm{a}}$ & 68 \\
\hline
\end{tabular}

B, Effect of KRAS status on PFS and OS

\begin{tabular}{|c|c|c|c|c|c|c|c|c|}
\hline & \multirow[b]{2}{*}{$\mathrm{n}$} & \multirow[b]{2}{*}{$\%$} & \multicolumn{3}{|c|}{ PFS } & \multicolumn{3}{|c|}{ OS } \\
\hline & & & $\begin{array}{l}\text { Median } \\
\text { (months) }\end{array}$ & $\operatorname{HR}(95 \% \mathrm{CI})$ & P-value & $\begin{array}{l}\text { Median } \\
\text { (months) }\end{array}$ & HR $(95 \%$ CI) & P-value \\
\hline \multicolumn{9}{|l|}{$K R A S$} \\
\hline G13D & 8 & 11 & 2.1 & & & 6.4 & & \\
\hline Other mutations & 13 & 20 & 1.7 & $1.52(0.95-2.59)$ & $0.074^{\mathrm{a}}$ & 5.2 & $1.18(0.67-2.28)$ & $0.577^{\mathrm{a}}$ \\
\hline Wild-type & 44 & 69 & 5.9 & $0.55(0.37-0.87)$ & $0.003^{\mathrm{a}}$ & 15.4 & $0.65(0.39-1.25)$ & $0.139^{\mathrm{a}}$ \\
\hline
\end{tabular}

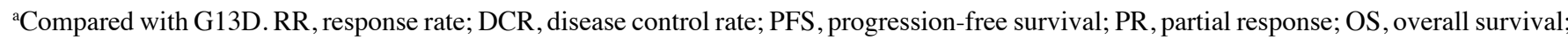
$\mathrm{SD}$, stable disease; PD, disease progression; HR, hazard ratio; CI, confidence interval; NA, not available.

Table III. Relationships between the KRAS mutation subtype and other molecular biomarkers.

\begin{tabular}{|c|c|c|c|c|c|}
\hline & \multirow[b]{2}{*}{$\mathrm{n}$} & \multirow[b]{2}{*}{$\%$} & \multicolumn{2}{|c|}{$K R A S$} & \multirow[b]{2}{*}{ P-value } \\
\hline & & & G13D & Other mutations & \\
\hline \multicolumn{6}{|l|}{$B R A F$} \\
\hline Wild-type & 21 & 100 & 8 & 13 & \\
\hline Mutant & 0 & 0 & 0 & 0 & Not available \\
\hline \multicolumn{6}{|l|}{ PIK3CA } \\
\hline Wild-type & 18 & 86 & 6 & 12 & \\
\hline Mutant & 3 & 14 & 2 & 1 & 0.531 \\
\hline \multicolumn{6}{|l|}{ PTEN } \\
\hline Normal expression & 12 & 57 & 5 & 7 & \\
\hline Loss of expression & 9 & 43 & 3 & 6 & 0.697 \\
\hline \multicolumn{6}{|l|}{ MET } \\
\hline Normal/low expression & 11 & 52 & 4 & 7 & \\
\hline Overexpression & 10 & 48 & 4 & 6 & 0.864 \\
\hline
\end{tabular}

2.0 months; HR, 1.61; 95\% CI, 0.98-2.69; P=0.044, Fig. 3C), although no significant association between levels of PTEN expression and RR, DCR or OS was detected in patients with mutant KRAS (Table IVA and B, Fig. 3D).

MET immunohistochemical evaluation. Overexpression of MET was detected in $10(48 \%)$ of the 21 patients with KRAS mutations. No significant correlation was found between MET expression status and the subtype of KRAS mutation
(Table III). MET overexpression was significantly associated with shorter PFS compared to normal MET expression (1.7 vs. 2.0 months; HR, 1.69; 95\% CI, 1.02-2.85; P=0.016, Fig. 3E), although no significant association between levels of MET expression and RR, DCR, or OS was noted in patients with mutant KRAS (Table IVA and B, Fig. 3F).

Multi-gene analysis. The results described above suggested that KRAS p.G13D mutation and normal PTEN and MET 
Table IV. Effect of biomarkers on RR, DCR, PFS and OS in patients with mutant KRAS.

A, Effect of biomarkers on RR and DCR

\begin{tabular}{|c|c|c|c|c|c|c|c|c|}
\hline & $\mathrm{n}$ & PR & $\mathrm{SD}$ & $\mathrm{PD}$ & $\mathrm{RR}(\%)$ & P-value & $\operatorname{DCR}(\%)$ & P-value \\
\hline \multicolumn{9}{|l|}{ PIK3CA } \\
\hline Wild-type & 18 & 0 & 3 & 15 & 0 & & 20 & \\
\hline Mutant & 3 & 0 & 0 & 3 & 0 & NA & 0 & 0.614 \\
\hline \multicolumn{9}{|l|}{ PTEN } \\
\hline Normal expression & 12 & 0 & 2 & 10 & 0 & & 17 & \\
\hline Loss of expression & 9 & 0 & 1 & 8 & 0 & NA & 11 & 0.612 \\
\hline \multicolumn{9}{|l|}{ MET } \\
\hline Normal/low expression & 11 & 0 & 2 & 9 & 0 & & 18 & \\
\hline Overexpression & 10 & 0 & 1 & 9 & 0 & NA & 10 & 0.538 \\
\hline
\end{tabular}

B, Effect of biomarkers on PFS and OS

\begin{tabular}{|c|c|c|c|c|c|c|c|c|}
\hline & \multirow[b]{2}{*}{$\mathrm{n}$} & \multirow[b]{2}{*}{$\%$} & \multicolumn{3}{|c|}{ PFS } & \multicolumn{3}{|c|}{ OS } \\
\hline & & & $\begin{array}{l}\text { Median } \\
\text { (months) }\end{array}$ & HR $(95 \%$ CI $)$ & P-value & $\begin{array}{l}\text { Median } \\
\text { (months) }\end{array}$ & $\mathrm{HR}(95 \% \mathrm{CI})$ & P-value \\
\hline \multicolumn{9}{|l|}{$P I K 3 C A$} \\
\hline Wild-type & 18 & 87.0 & 1.8 & & & 5.2 & & \\
\hline Mutant & 3 & 13.0 & 2.0 & $0.91(0.44-1.58)$ & 0.757 & 6.4 & $0.52(0.12-1.19)$ & 0.187 \\
\hline \multicolumn{9}{|l|}{ PTEN } \\
\hline Normal expression & 12 & 57.1 & 2.0 & & & 6.4 & & \\
\hline Loss of expression & 9 & 42.9 & 1.7 & $1.62(0.98-2.69)$ & 0.044 & 5.2 & $0.98(0.56-1.63)$ & 0.927 \\
\hline \multicolumn{9}{|l|}{ MET } \\
\hline Normal/low expression & 11 & 52.3 & 2.0 & & & 7.1 & & \\
\hline Overexpression & 10 & 47.7 & 1.7 & $1.69(1.02-2.85)$ & 0.016 & 5.1 & $1.34(078-2.30)$ & 0.186 \\
\hline
\end{tabular}

RR, response rate; DCR, disease control rate; PFS, progression-free survival; OS, overall survival; PR, partial response; SD, stable disease; $\mathrm{PD}$, disease progression; HR, hazard ratio; CI, confidence interval; NA, not available.

expression may be potentially favorable prognostic factors in mCRCs with KRAS mutations treated by anti-EGFR MoAbs. To test whether incorporation of multi-gene profiles is useful for the prediction of the response to anti-EGFR therapies, we further analyzed the patients having multiple favorable factors. There were 2 patients with KRAS p.G13D mutations showing no abnormality in PTEN and MET expression. Both patients exhibited stable disease to anti-EGFR therapies, and PFS of these two patients (5.3 and 4.8 months) were the first and second longest among the 21 patients with KRAS mutations (range of other 19 patients, 0.7-3.7 months). The PFS of these 2 patients were similar to the PFS of patients with wild-type $K R A S$ (median; 5.9 months, 95\% CI, 3.5-7.3 months).

\section{Discussion}

Since anti-EGFR MoAb therapies are currently restricted to patients with wild-type $K R A S$ in many countries, few data are available regarding the response to these therapies in $\mathrm{mCRC}$ patients with mutant KRAS. To the best of our knowledge, the research presented here represents the first study to analyze associations among subtypes of $K R A S$, additional genetic alterations, and efficacy of anti-EGFR MoAb therapies in mCRC patients with KRAS mutations.

The most striking finding in this study was the fact that loss of PTEN expression and MET overexpression was associated with reduced PFS even in patients with KRAS mutations. PTEN is a tumor-suppressor protein that regulates the PI3K/ Akt signal transduction pathway. Loss of PTEN production is associated with intrinsic activation of the Akt pathway, conferring resistance to inhibitors of the HER family (29). Accordingly, low PTEN expression has been associated with lack of response to anti-EGFR MoAbs in several reports $(4,5)$, although such a correlation was not reported by other researchers $(16,18)$. In these earlier analyses, the presence of $K R A S$ mutations was not specified, precluding recognition of such an association in patients with $K R A S$-mutated mCRCs.

MET is an oncogene that contains a tyrosine kinase domain and can activate the RAS/RAF/MAPK and PTEN/PI3K/Akt pathways by itself or via EGFR transphosphorylation $(19,30)$. 
MET reportedly is involved in many mechanisms of cancer proliferation and metastasis. MET additionally contributes to cancer resistance to EGFR inhibitors through bypass signaling. In CRC, overexpression of MET has been suggested to be associated with tumor progression $(31,32)$. Subsequent studies have indicated an association of MET overexpression with poor outcome of mCRCs, including inferior response to anti-EGFR therapy (18). However, as with PTEN above, these studies did not separately analyze patients with KRAS mutations.

Our present data regarding PTEN and MET indicate that mCRC patients lacking MET overexpression or loss of PTEN may show some benefit by these therapies even if these patients harbor KRAS mutations in their tumors. DCR also was inferior in patients with these molecular aberrations, although the difference fell short of statistical significance. These results suggest that the policies restricting anti-EGFR MoAb therapies to mCRC patients with wild-type KRAS might deprive some patients who would benefit from such treatments. Assessment of genetic alterations in EGFR signaling pathways other than $K R A S$ may enable physicians to identify patients who would benefit from anti-EGFR treatment despite the presence of KRAS mutations.

Several lines of evidence also indicate that $K R A S$ p.G13Dmutated CRC defines a less aggressive phenotype and is more sensitive to anti-EGFR treatment than codon-12 mutated CRC. While mutations in either codons 12 or 13 affect the intrinsic GTPase activity of the KRAS protein, structural and functional analyses demonstrated that the glycine residue at position 12 is more critical for function of wild-type KRAS than the glycine at position 13 (33). In addition, transfection of NIH3T3 cells with KRAS mutated in codon 12 resulted in a more aggressive phenotype than that observed in cells transfected with KRAS harboring the codon-13 mutation (11). In accordance with these observations, several clinical studies have revealed that the tumors from patients who responded to anti-EGFR therapies predominantly harbored codon-13 mutations, and all codon-13 mutant responders carried the p.G13D mutation (11-13). More importantly, 3 meta-analyses have indicated that patients with the p.G13D mutation who received cetuximab showed a better response to cetuximab than patients with $K R A S$ codon-12 mutations $(10,12,34)$. In these analyses, the p.G13D mutation was associated with longer PFS and OS of patients treated with a combination of cetuximab plus chemotherapy compared to other KRAS mutations. The present study is, to our knowledge, the first study conducted in a single institute to clarify the association of the p.G13D mutation with superior clinical response (DCR) to anti-EGFR MoAbs in mCRC patients. In fact, patients with the p.G13D mutation exhibited DCR equivalent to those with wild-type $K R A S$. Lack of statistical significance in the prolonging of PFS in patients with p.G13D may be due in part to the small sample size in our study. Although meta-analysis is generally considered to provide higher levels of confidence than single studies, the present study (involving patients treated by relatively uniform therapies in a single institute) reinforces the results observed in meta-analyses. These observations should prompt further clinical trials to investigate whether mCRC patients with p.G13D might benefit from anti-EGFR MoAb therapies.

In testing molecular biomarkers as predictors of therapeutic responses, multi-gene models have been proposed to be supe- rior to single-gene models $(5,16)$. The present study could not precisely analyze the feasibility of multi-gene models, because the sample size was small. However, as described above, incorporation of multiple factors (p.G13D, normal PTEN and MET expression) appeared to identify patients who showed the best response to anti-EGFR therapies. These results suggest that PTEN and MET expression levels, together with subtype of $K R A S$ mutation, might be candidate criteria for the selection of $K R A S$-mutant patients expected to exhibit favorable responses to anti-EGFR MoAbs. The incorporation of PTEN, MET and subtypes of KRAS mutation into the design of clinical trials may permit further individualization of treatment for $\mathrm{mCRC}$ by helping to define true predictive markers (20). If these data are confirmed in future large studies, KRAS-mutated mCRC patients having these molecular features might be eligible for anti-EGFR MoAb therapy before trials of treatment by novel therapeutic drugs for $\mathrm{mCRC}$, such as regorafenib or aflibercept $(35,36)$.

The present study has some limitations. Notably, our study was performed retrospectively in a relatively small and heterogeneous population. The small sample size may have contributed to lack of statistical significance in some analyses. The majority of our population $(90 \%)$ was treated with two or more chemotherapy regimens before anti-EGFR MoAb therapy. Additionally, the anti-EGFR treatment protocols were heterogeneous. Our findings therefore require further validation in subsequent prospective studies prior to application in the clinical practice.

In conclusion, our data demonstrated the potential utility of alterations in PTEN and MET expression as predictive markers for response to anti-EGFR MoAbs in mCRC patients with $K R A S$ mutations. In addition, we confirmed the predictive value of the KRAS p.G13D mutation for better response to anti-EGFR therapies in comparison with other KRAS mutations. Thus, a subset of mCRC patients with KRAS mutations who harbor specific additional molecular features exhibited responsiveness to anti-EGFR MoAb treatment that was equivalent to that of patients with wild-type $K R A S$. These results warrant further studies re-examining the current policy restricting anti-EFGR therapies to patients with wild-type KRAS.

\section{References}

1. Linardou H, Dahabreh IJ, Kanaloupiti D, et al: Assessment of somatic k-RAS mutations as a mechanism associated with resistance to EGFR-targeted agents: a systematic review and meta-analysis of studies in advanced non-small-cell lung cancer and metastatic colorectal cancer. Lancet Oncol 9: 962-972, 2008.

2. Normanno N, Tejpar S, Morgillo F, De Luca A, Van Cutsem E and Ciardiello F: Implications for KRAS status and EGFRtargeted therapies in metastatic CRC. Nat Rev Clin Oncol 6: 519-527, 2009.

3. Messner I, Cadeddu G, Huckenbeck W, Knowles HJ, Gabbert HE, Baldus SE and Schaefer KL: KRAS p.G13D mutations are associated with sensitivity to anti-EGFR antibody treatment in colorectal cancer cell lines. J Cancer Res Clin Oncol 139: 201-209, 2013.

4. Loupakis F, Pollina L, Stasi I, et al: PTEN expression and KRAS mutations on primary tumors and metastases in the prediction of benefit from cetuximab plus irinotecan for patients with metastatic colorectal cancer. J Clin Oncol 27: 2622-2629, 2009.

5. Saridaki Z, Tzardi M, Papadaki C, et al: Impact of $K R A S, B R A F$, PIK3CA mutations, PTEN, AREG, EREG expression and skin rash in $\geq 2$ line cetuximab-based therapy of colorectal cancer patients. PLoS One 6: e15980, 2011. 
6. O'Neil BH: Systemic therapy for colorectal cancer: focus on newer chemotherapy and novel agents. Semin Radiat Oncol 13: 441-453, 2003.

7. Adlard JW, Richman SD, Seymour MT and Quirke P: Prediction of the response of colorectal cancer to systemic therapy. Lancet Oncol 3: 75-82, 2002.

8. Benvenuti S, Sartore-Bianchi A, Di Nicolantonio F, et al: Oncogenic activation of the RAS/RAF signaling pathway impairs the response of metastatic colorectal cancers to antiepidermal growth factor receptor antibody therapies. Cancer Res 67: 2643-2648, 2007.

9. Bando H, Yoshino T, Yuki S, et al: Clinical outcome of Japanese metastatic colorectal cancer patients harbouring the KRAS p.G13D mutation treated with cetuximab + irinotecan. Jpn J Clin Oncol 42: 1146-1151,2012.

10. Tejpar S, Celik I, Schlichting M, Sartorius U, Bokemeyer C and Van Cutsem E: Association of KRAS G13D tumor mutations with outcome in patients with metastatic colorectal cancer treated with first-line chemotherapy with or without cetuximab. J Clin Oncol 30: 3570-3577, 2012.

11. Guerrero S, Casanova I, Farré L, Mazo A, Capellà G and Mangues R: K-ras codon 12 mutation induces higher level of resistance to apoptosis and predisposition to anchorageindependent growth than codon 13 mutation or proto-oncogene overexpression. Cancer Res 60: 6750-6756, 2000.

12. De Roock W, Jonker DJ, Di Nicolantonio F, et al: Association of KRAS p.G13D mutation with outcome in patients with chemotherapy-refractory metastatic colorectal cancer treated with cetuximab. JAMA 304: 1812-1820, 2010.

13. Peeters M, Douillard JY, Van Cutsem E, Siena S, Zhang K Williams R and Wiezorek J: Mutant KRAS codon 12 and 13 alleles in patients with metastatic colorectal cancer: assessment as prognostic and predictive biomarkers of response to panitumumab. J Clin Oncol 31: 759-765, 2013.

14. Gajate P, Sastre J, Bando I, et al: Influence of KRAS p.G13D mutation in patients with metastatic colorectal cancer treated with cetuximab. Clin Colorectal Cancer 11: 291-296, 2012.

15. Di Nicolantonio F, Martini M, Molinari F, et al: Wild-type $B R A F$ is required for response to panitumumab or cetuximab in metastatic colorectal cancer. J Clin Oncol 26: 5705-5712, 2008.

16. Ulivi P, Capelli L, Valgiusti M, et al: Predictive role of multiple gene alterations in response to cetuximab in metastatic colorectal cancer: a single center study. J Transl Med 10: 87, 2012.

17. Kato S, Iida S, Higuchi T, et al: PIK3CA mutation is predictive of poor survival in patients with colorectal cancer. Int J Cance 121: 1771-1778, 2007

18. Inno A, Di Salvatore M, Cenci T, et al: Is there a role for IGF1R and c-MET pathways in resistance to cetuximab in metastatic colorectal cancer? Clin Colorectal Cancer 10: 325-332, 2011.

19. Guo A, Villén J, Kornhauser J, et al: Signaling networks assembled by oncogenic EGFR and c-Met. Proc Natl Acad Sci USA 105: 692-697, 2008

20. Sattler M, Reddy MM, Hasina R, Gangadhar T and Salgia R: The role of the c-Met pathway in lung cancer and the potential for targeted therapy. Ther Adv Med Oncol 3: 171-184, 2011.

21. Therasse P, Arbuck SG, Eisenhauer EA, et al: New guidelines to evaluate the response to treatment in solid tumors. European Organization for Research and Treatment of Cancer, National Cancer Institute of the United States, National Cancer Institute of Canada. J Natl Cancer Inst 92: 205-216, 2000
22. Ohnishi H, Ohtsuka K, Ooide A, Matsushima S, Goya T and Watanabe T: A simple and sensitive method for detecting major mutations within the tyrosine kinase domain of the epidermal growth factor receptor gene in non-small-cell lung carcinoma. Diagn Mol Pathol 15: 101-108, 2006.

23. Torres J, Navarro S, Roglá I, et al: Heterogeneous lack of expression of the tumour suppressor PTEN protein in human neoplastic tissues. Eur J Cancer 37: 114-121, 2001.

24. Dua R, Zhang J, Parry G and Penuel E: Detection of hepatocyte growth factor (HGF) ligand-c-MET receptor activation in formalin-fixed paraffin embedded specimens by a novel proximity assay. PLoS One 6: e15932, 2011.

25. Murray S, Karavasilis V, Bobos M, et al: Molecular predictors of response to tyrosine kinase inhibitors in patients with non-smallcell lung cancer. J Exp Clin Cancer Res 31: 77, 2012.

26. Lin B, Utleg AG, Gravdal K, et al: WDR19 expression is increased in prostate cancer compared with normal cells, but low-intensity expression in cancers is associated with shorter time to biochemical failures and local recurrence. Clin Cancer Res 14: 1397-1406, 2008.

27. Halvorsen OJ, Rostad K, Øyan AM, et al: Increased expression of SIM2-s protein is a novel marker of aggressive prostate cancer. Clin Cancer Res 13: 892-897, 2007.

28. Zeng ZL, Wu WJ, Yang J, et al: Prognostic relevance of melanoma antigen D1 expression in colorectal carcinoma. J Transl Med 10: $181,2012$.

29. Pandolfi PP: Breast cancer - loss of PTEN predicts resistance to treatment. N Engl J Med 351: 2337-2338, 2004

30. Jo M, Stolz DB, Esplen JE, Dorko K, Michalopoulos GK and Strom SC: Cross-talk between epidermal growth factor receptor and c-Met signal pathways in transformed cells. J Biol Chem 275: 8806-8811, 2000

31. Takeuchi H, Bilchik A, Saha S, et al: c-MET expression level in primary colon cancer: a predictor of tumor invasion and lymph node metastases. Clin Cancer Res 9: 1480-1488, 2003.

32. Trusolino L and Comoglio PM: Scatter-factor and semaphorin receptors: cell signalling for invasive growth. Nat Rev Cancer 2: 289-300, 2002

33. Kiaris H and Spandidos DA: Mutations of ras genes in human tumours (Review). Int J Oncol 7: 413-421, 1995.

34. Mao C, Huang YF, Yang ZY, Zheng DY, Chen JZ and Tang JL: KRAS p.G13D mutation and codon 12 mutations are not created equal in predicting clinical outcomes of cetuximab in metastatic colorectal cancer: A systematic review and meta-analysis. Cancer 119: 714-721, 2013

35. Strumberg D, Scheulen ME, Schultheis B, et al: Regorafenib (BAY 73-4506) in advanced colorectal cancer: a phase I study. Br J Cancer 106: 1722-1727, 2012.

36. Van Cutsem E, Tabernero J, Lakomy R, et al: Addition of aflibercept to fluorouracil, leucovorin, and irinotecan improves survival in a phase III randomized trial in patients with metastatic colorectal cancer previously treated with an oxaliplatin-based regimen. J Clin Oncol 30: 3499-3506, 2012. 